\title{
Filariose linfática: doença potencialmente eliminável
}

\author{
Lymphatic filariasis: \\ a potentially eradicable disease
}

Gerusa Dreyer 1

Giovanini Coelho 2

\footnotetext{
1 Departamento de Parasitologia, Centro de Pesquisas Aggeu Magalhães, Fundação Oswaldo Cruz. Av. Moraes Rego s/no, Cidade Universitária, Recife, PE 50670-420, Brasil.

2 Fundação Nacional de Saúde, Setor de Autarquias Sul. Quadra 4, Bloco N, 7 o andar, Brasília, DF 70058-902, Brasil.
}

\begin{abstract}
The recent demonstration that single-dose ivermectin, diethylcarbamazine, or a combination of these drugs can profoundly suppress Wuchereria bancrofti and Brugia malayi microfilaremia for periods of six months to two years has led to renewed hope that transmission can be interrupted and lymphati c filariasis eradicated. Based in part on the availability of these new chemotherapeutic tools, the International Task Force for Disease Eradication recently identified lymphatic filariasis as one of the few diseases that could potentially be eradicated. Thus, control programs based on mass treatment (whether supplemented or not by vector control) have begun to be implemented in some endemic areas. We provide a brief review of available anti-filarial drugs for use in humans, including their tolerance and efficacy.

Key words Lymphatic Filariosis; Control; Ivermectin; Diethylcarbamazine; Wuchereria bancrofti
\end{abstract}

Resumo Os resultados obti dos com o uso de esquemas terapêuti cos simples, como dose única anual ou bianual de Ivermectina (IV), Dietilcarbamazina (DEC) sozinhas ou combinadas, têm sido surpreendentemente promissores na redução da infecção linfática causada pela Wuchereria bancrofti eBrugia malayi. Assim, perspectivas existem de eliminar a doença dos países endêmicos, se programas de controle forem empregados usando-se o tratamento em massa, complementado ou não pelo controle do vetor. Uma breve revisão éfeita sobre cada droga em relação à eficácia e às reações adversas causadas pela morte dos di versos estági os do parasi ta no homem infectado.

Palavras-chave Filariose Linfática; Controle; Ivermectina; Dietilcarbamazina; Wuchereria bancrofti 


\section{Introdução}

A prevalência da filariose linfática vem aumentando em países de clima tropical e subtropical, principalmente pela expansão de urbanização não planejada em muitas áreas endêmicas. Estima-se que cerca de 120 milhões de pessoas estejam infectadas com Wuchereria bancrofti e oito milhões com Brugia malayi. Muitos desses indivíduos exibem formas clínicas da doença, sendo o linfedema (tanto em áreas de W. bancrofti como de Brugia malayi) e a hidrocele (somente em bancroftose) as manifestações mais prevalentes (WHO, 1994). O mais surpreendente, e só reconhecido recentemente, é que os indivíduos infectados mas que não mostram morbidade clínica já apresentam formas subclínicas, representadas principalmente pela hematúria e linfangiectasia (Dreyer et al., 1992; Norões et al., 1996b). Os resultados obtidos com o uso de esquemas terapêuticos simples, como dose única anual ou bianual de Ivermectina (Iv), Dietilcarbamazina (DEC), sozinhas ou combinadas (WHO, 1994), ou mesmo o consumo diário do sal de cozinha enriquecido com a DEC têm sido surpreendentemente promissores na redução da infecção (Gelband, 1994). Essa última estratégia de controle não será abordada neste trabalho, pelas peculiaridades de adesão de seu uso em tratamento em massa, restritas a poucas regiões do mundo.

A disponibilidade de novas estratégias de controle, em conjunto com observação feita em 1993 pela Força Tarefa Internacional para Erradicação de Doenças (CDC, 1993) de que a filariose linfática seria uma das seis doenças infecciosas consideradas erradicáveis ou potencialmente erradicáveis, fez deste um momento ímpar para se propor e se iniciar programas de controle, com um potencial de eliminação da doença nos países endêmicos.

Ratificando e tornando ainda mais realístico o que foi dito acima, anuncia-se em 1996 (TDR, 1996) que quatro das oitos doenças que recebem suporte do "Special Programme for Research and Training in Tropical Disease TDR" seriam alvo de eliminação a partir das seguintes estratégias:

1) Hanseníase: usando-se a multidrogaterapia.

2) Oncocercose: usando-se a ivermectina.

3) Filariose linfática: usando-se a dietilcarbamazina e/ ou a ivermectina.

4) Doença de Chagas: uso racional de inseticida e controle em banco de sangue.

\section{Considerações gerais}

O tratamento em massa, preconizado atualmente para controle/ eliminação da infecção, exclui o diagnóstico parasitológico, impedindo assim a identificação do(s) parasito(s) do(s) qual (quais) o indivíduo é portador. Existem na África muitas áreas en dêmicas onde Onchocerca volvulus ou Loa loa co-existem com W. bancrofti no mesmo indivíduo. A dietilcarbamazina (DEC) está proscrita em áreas de oncocercose e tem seu uso muito limitado, em nível individual, em loíase (WHO, 1994). Assim, a ivermectina, que é a droga de escolha para o tratamento da oncocercose e tem uma potente ação microfilaricida na bancroftose (Chodakewitz, 1995), poderá ser usada como droga de eleição sem restrições geográficas; universaliza-se assim, um esquema posológico para o controle da filariose linfática. Por outro lado, o uso da DEC, sozinha ou em associação com a Iv, ficaria então a critério de cada região em que não co-existisse a oncocercose ou a loíase.

Em áreas de transmissão potencial ou naquelas em que a prevalência da infecção é desconhecida, a pesquisa do parasita no humano (Zhong et al., 1996) e/ ou no mosquito (Chanteau et al., 1994) deve constituir-se como uma etapa necessária para se saber se um controle deve ser iniciado. Em áreas sabidamente endêmicas, o conhecimento da situação real em termos de transmissibilidade, principalmente da prevalência e da extensão da endemia, é um parâmetro que deve estar igualmente disponível. Assim, poderá ser determinada a área a ser coberta, quão efetivo o controle está sendo e quando este deve ser descontinuado. A interrupção precoce das campanhas de controle tem sido a principal causa do fracasso no controle da filariose linfática, assim como de muitas outras doenças, principalmente as parasitárias.

Inquéritos "clínicos rápidos" também seriam opções para se determinar a ocorrência e talvez até se estimar a prevalência da infecção em uma dada região. Procedimentos, tais como: levantamento retrospectivo de prontuários clínicos de hospitais de referência ou exame físico, na comunidade, de indivíduos do sexo masculino, identificando-se o portador de hidrocele, e o uso da ultra-sonografia para se detectar o portador de vermes adultos vivos de W. bancrofti (Norões et al., 1996a, 1996b) poderiam ser opções factíveis para muitas regiões.

Existe uma tentativa de se substituir a coleta de sangue noturna pela diurna para se determinar o nível de endemicidade de uma determinada área. A avaliação da antigenemia 
durante o dia em amostras de sangue capilar tem se mostrado como uma alternativa para substituir os inquéritos noturnos (WHO, 1994). O diagnóstico da infeção no mosquito vetor com sondas de DNA tem também se mostrado muito promissor (Chanteau et al., 1994)

Modelos matemáticos têm fornecido adicionalmente uma ajuda para se prever e avaliar estratégias de controle em outras doenças parasitárias (Chan, 1995; Plaisier et al., 1990). Tais modelos podem ser de valor particular em filariose linfática por causa da complexidade de interações entre o vetor, o humano e o parasito. É importante ressaltar que o custo econômico de cada estratégia está sendo incorporado à simulação matemática, o que permite uma estimativa do custo, adequada para a realidade de cada região.

De forma sintética, são os seguintes alvos que devem ser considerados no controle da fiIariose linfática:

Controle da infecção (transmissibilidade)

São duas as estratégias gerais (as quais não precisam ser mutuamente exclusivas), consideradas para reduzir a transmissão da infecção filarial:

a) tratar a população humana, o que fará descrescer a microfilaremia;

b) diminuir o contato homem-vetor, usualmente pela redução da densidade de mosquitos transmissores.

Controle da doença (morbidade)

Recentes avanços no entendimento da patogênese da filariose linfática trouxeram à tona o reconhecimento da importância da infecção secundária na gênese da elefantíase (Dreyer et al., no prelo). O episódio agudo bacteriano, exacerbaria a disfunção linfática, por sua vez provocada diretamente pelo parasita filarial, promovendo a evolução para edemas persistentes e severos até a forma mais grave, conhecida como elefantíase (WHO, 1994). Foi possível também se detectar a existência e a extensão de doença subclínica filarial nos portadores “assintomáticos" de microfilaremia (Dreyer et al., 1992; Norões et al., 1996b), podendo-se dessa maneira criar um programa preventivo e evitar a evolução para algumas das formas crônicas.

\section{Quimioterapia}

$\underline{\text { Ivermectina (Iv) }}$

A Iv é um componente dos parasiticidas conhecidos como avermectina/milbencinina. Foi registrada em 1981 e tem sido aprovada para uso em mais de 60 países para controlar para- sitoses em animais e, mais recentemente, também para seres humanos. Para o uso humano está disponível em forma de comprimidos de $6 \mathrm{mg}$ e tem o nome comercial de Mectizan, (Merck, Sharp \& Dhome). O emprego dessa droga em populações humanas foi um marco sem precedentes no controle da oncocercose (ou doença da cegueira dos rios, como também é conhecida). Vem sendo usada em tratamento em massa para essa doença desde 1987, na dose de 150-200 microgramas/ kg, em esquema anual. Após a ingesta da droga observa-se uma rápida eliminação da microfilária de Oncocerca volvulus da pele, assim como a sua redução gradual em região ocular. As reações adversas causadas pela morte das microfilárias são geralmente leves.

A Iv é uma potente lactona monocíclica, produzida pelo Streptomyces avermitilis, que causa paralisia em muitos nematódeos e artrópodes. Tem mostrado uma boa ação microfilaricida também em alguns outros filarídeos que infectam o homem (W. bancrofti, B. malayi, Loa loa e Mansonella ozzardi), porém é inativa contra a Mansonella perstans. Por outro lado, tem sido aparentemente ineficaz contra os vermes adultos (Dreyer et al., 1995b, 1995c; Coutinho et al., 1994; Dreyer et al., 1996) dos supracitados parasitos. Tem uma boa ação contra Strongyloi des stercoralis, Ascaris lumbricoi des, Trichuris trichiura, Enterobius vermi cularese Larva mi grans. Tem pouca ou nenhuma atividade contra Ancilostomídeos. Estudos preliminares também indicam que a I v tem a potencialidade de se tornar a droga de escol ha para tratar ecto-parasitas em seres humanos (Kar et al., 1994). Ampla revisão sobre essa nova droga foi feita pelo Ottesen \& Campbell (1994), abrangendo farmacologia e modo de ação, estudos clínicos, ação nas diversas filárias e em parasitos intestinais.

Quando administrada em pacientes microfilarêmicos infectados pelo parasito filarial que habita os vasos linfáticos, provoca reação sistêmica, geralmente leve, em parte dos indivíduos, sendo os sintomas/sinais mais comuns a febre, a cefaléia, e a mialgia. A severidade da reação sistêmica depende da densidade de microfilaremia, sendo mais acentuada naqueles indivíduos portadores de altas parasitemias. A reação, por sua vez, é autolimitada, com duração de cerca de 48 horas e só ocorre praticamente na primeira tomada da droga (Coutinho et al., 1994). A baixa considerável no número dos parasitos circulantes induzida pelo primeiro ciclo de tratamento faz com que as tomadas subseqüentes não tenham praticamente nenhuma repercussão clínica para o indivíduo. 
No tratamento em massa ou individual, nenhuma reação é vista a partir da terceira tomada da droga, mesmo em programas com interval os anuais. Esse fato é desejável em programas de controle de longa duração, como é o caso da filariose bancroftiana. Por outro lado, como não parece ter efeito macrofilaricida (Dreyer et al., 1995c; Dreyer et al., 1996) , não causa reação localizada no sistema linfático (linfangites, adenites, epididimites ou funiculites). Apesar de parecer uma limitação o fato de a Iv não matar os vermes adultos, reveste-se de grande importância o seu emprego em áreas onde a DEC não é aceita, exatamente por desencadear as reações adversas do tipo localizadas (Sutanto et al., 1985; Cartel et al., 1992). A Iv tem sido amplamente estudada na filariose linfática, quer no tratamento em massa quer no seletivo (Moulia-Pelat et al., 1994; Zheng et al., 1991; Coutinho et al., 1994), e estudos empregando-se as diversas doses mostraram que 400 microgramas/ kg foi a melhor posologia em termos de clearance de microfilárias obtida a longo prazo, com tolerância equival ente a doses mais baixas (Coutinho et al., 1994; Dreyer et al., 1995b; Richards et al., 1991; Addiss et al., 1993).

\section{Dietilcarmamazina (DEC)}

Há quase cinqüenta anos, a DEC é a droga de escolha para o tratamento da filariose bancroftiana, mesmo não se conhecendo aspectos fundamentais, como por exemplo seu mecanismo de ação ou mesmo a posologia ideal para os pacientes portadores das diversas formas clínicas da doença. Até o momento, é também a única droga a ter efeito comprovado sobre o verme adulto de Wuchereria bancrofti (Ottesen, 1985; Dreyer et al., 1995a, 1995b; Dreyer et al., 1994; Figueredo-Silva et al., 1996; Norões et al., 1997). A dose preconizada pela OMS para o tratamento individual é de $6 \mathrm{mg} / \mathrm{kg} /$ dia por 12 dias, podendo ser repetida se houver persistência da parasitemia ou sintoma clínico (WHO, 1992). Entretanto, estudos parasitológicos relativamente recentes têm trazido novos conhecimentos sobre a eficácia da DEC em dose única, o que a faz promissora novamente para uso em nível de comunidade (Cartel et al., 1990).

Andrade et al. (1995) mostraram que em dose única de $6 \mathrm{mg} / \mathrm{kg}$ o efeito microfilaricidada da DEC a longo prazo é similar ao do tratamento de 12 dias supracitado. Isso ratificou a inclusão da DEC no tratamento em massa para o controle da filariose em áreas onde não existe restrição de seu uso. Um estudo cuidadoso sobre a tolerância à DEC em diversas doses foi feito em Recife-PE (Dreyer et al., 1994), incluindo indivíduos infectados assintomáticos, estes apresentando doença clínica, assim como indivíduos considerados endêmicos normais. Os resultados indicaram a boa tolerabilidade da DEC nos diversos grupos tratados; vale salientar a ocorrência de reação localizada em um indivíduo do grupo considerado normal, sinalizando que o mesmo estava infectado, embora não apresentasse microfilaremia ou sintomas clínicos da doença. Esse fato ratifica a importância do tratamento em massa, para identificar aqueles indivíduos que não seriam diagnosticados no exame seletivo.

Pelo fato de ter a DEC um efeito microfilaricida menos potente que a IV, os indivíduos tratados geral mente tendem a apresentar sintomatologia sistêmica um pouco mais leve. Por outro lado, como tem ação adulticida, ao contrário da ivermectina, os efeitos localizados da morte do verme causam linfangites, adenites e sintomatologia aguda urológica, inclusive a hidrocele. Hoje, após o advento da ultra-sonografia, é possível monitorizar in vivo com precisão o efeito de drogas sobre o verme adulto de W. bancrofti (Dreyer et al., 1995a, 1995c; Dreyer et al., 1996; Norões et al., 1997). Mostrou-se que, embora doses únicas de $1 \mathrm{mg} / \mathrm{kg}$ sejam também capazes de matar o verme adulto (Figueredo-Silva et al., 1996), há uma diferença significativa quando doses únicas de 6 ou $12 \mathrm{mg} / \mathrm{kg}$ são usadas (Norões et al., 1997). Por outro lado, a DEC não mata todos os vermes adultos em um determinado indivíduo. Duas subpopulações parecem existir: uma tolerante e outra sensível, com insensibilidade aparentemente de 50\%. Espera-se que essa insensibilidade à DEC seja dependente da idade e que o verme se torne sensível à droga, à medida que envelhece. Assim, para programas de controle, os achados supracitados respaldam plenamente a preconização feita pela OMS para o uso da DEC em dose única de $6 \mathrm{mg} / \mathrm{kg}$ em regimes semestrais, anuais ou bianuais (WHO, 1994).

Combinação Ivermectina e Dietilcarbamazina

A multiterapia é hoje uma opção em muitas doenças, como hanseníase e AIDS, por exemplo. Como se dispõe de uma droga com excelente efeito microfilaricida e outra com propriedade adulticida, por que não combinar as duas? Em relação à persistência da redução da microfilaremia, os resultados do esquema combinado foram bem mais promissores do que quando se usaram as drogas isoladamente (Coutinho et al., 1994; Dreyer et al., 1995b; Ad- 
diss et al., 1993), o que fez desse esquema um forte candidato para ser empregado na interrupção da transmissibilidade (Moulia-Pelat et al., 1995a, 1995b). Apesar de as duas drogas já estarem liberadas para serem administradas simultaneamente do ponto de vista de interação medicamentosa (WHO, 1994), só se conhece atualmente o efeito potenciador que existe no clearance da microfilaremia. Ainda não se pode precisar se o efeito macrofilaricida da DEC será mantido ou se a interação torna a Iv uma droga macrofilaricida. Para resolver a questão, um estudo ultra-sonográfico, financiado pela OMS, já foi iniciado em Recife-Brasil e seus resultados estarão brevemente disponíveis.

Os conhecimentos acumulados sobre a eficácia da quimioterapia na filariose linfática deverão alimentar os modelos matemáticos que, por sua vez, deverão ter a capacidade de prever a duração de programas de controle. Isso se reveste de muita importância quando a totalidade ou parte da população adulta parasitária não é morta pelo tratamento empregado, uma vez que a vida média reprodutiva do verme adulto fêmea é estimada em cerca de 8 a 10 anos (Venamail et al., 1989).

\section{Controle do vetor}

Este é um tema bastante complexo, pois depende não só da disponibilidade de novas ferramentas, como por exemplo o controle biológico pelo Bacillus sphaericus (Hougard et al., 1993; Cheong \& Yap, 1985; Regis et al., 1995), mas também do número de criadouros e da velocidade de aparecimento de novos focos, dos recursos financeiros e da infra-estrutura peculiares a cada região endêmica. Por outro lado, o controle de vetor normalmente propicia oportunidade de aumento de adesão da população ao controle da doença e tem tido um papel importante no controle da filariose em muitos locais. Além do mais, a diminuição da densidade do vetor tem contribuído também para a manutenção da interrupção da transmissão em algumas áreas (WHO, 1994). Entretanto, programas de controle baseados exclusivamente na redução total ou parcial do vetor raramente têm sido mantidos suficientemente para decrescer a prevalência da infecção filarial em populações humanas. A experiência mundial sugere que o controle de vetor deve ser sempre complementar ao tratamento quimioterápico, pela dificuldade de manutenção do mesmo por longos períodos, principalmente pelo seu alto custo no orçamento global das regiões (WHO, 1994). Indiscutivelmente, o saneamento básico, em áreas onde o vetor é o Culex quinquefasciatus, seria a solução permanente para a interrupção da transmissibilidade, a exemplo do que foi feito no Japão (Sasa, 1976).

\section{Comentário final}

Embora os métodos necessários para controlar/ eliminar a filariose linfática estejam disponíveis, é necessário desenvolver e avaliar estratégias de implementações apropriadas, que deverão não só ser factíveis economicamente, mas também aceitas pelas comunidades e mantidas a longo prazo. Assim, a(s) estratégia(s) ótima(s) difere $(m)$ de região para região, devendo-se levar em conta as particularidades de cada área endêmica associadas à infra-estrutura para a saúde pré-existente (agentes comunitários ou de saúde, por exemplo) e aos aspectos culturais também peculiares a cada região. Como a ivermectina ainda não está registrada para o tratamento da filariose linfática pelo laboratório fabricante, esse fato poderá retardar o emprego da mesma em alguns países, como o Brasil, por exemplo. No entanto, em outros países, como o Egito e Samoa, programa de controle nacional usando a ivermectina já foi deflagrado. Os estudos individuais ou em comunidades já disponíveis na literatura respaldam o importante papel da ivermectina e da DEC em quebrar a transmissibilidade da filariose linfática, principalmente da bancroftiana, tornando-a assim uma doença eliminável. 
ADDISS, D. G.; EBERHARD, M. L.; LAMMIE, P. J.; MCNEELY, M. B.; LEE, S. H.; MCNEELY, D. F. \& SPENCER H. C., 1993. Comparative efficacy of clearing-dose and single high-dose ivermectin and diethylcarbamazine againt Wuchereria bancrofti microfilaremia. American Journal of Tropical Medicineand Hygiene, 48:178-185.

ANDRADE, L. D.; MEDEIROS, Z.; PIRES, M. L.; PIMENTEL, A.; ROCHA, A.; FIGUEREDO-SILVA, J.; COUTINHO, A. \& DREYER, G., 1995. Comparative efficacy of three different diethylcarbamazine regimens in lymphatic filariasis. Transactions of the Royal Society of Tropical Medicine and Hygiene, 89:319-321.

CARTEL, J. L.; CELERIER, P.; SPINEGEL, A.; BURUCOA, C. \& ROUX, J.F., 1990. A single diethylcarbamazine dose for treatment of Wuchereria bancrofti carriers in French Polynesia: efficacy and side effects. Southeast Asian Journal of Tropical Medicineand Public Health, 21:465-470.

CARTEL, J. L.; SPIEGEL, N. N. L.; CARDINES, R.; PLICHART, R.; MARTIN, P. M.; ROUX, J. F. \& MOULIA-PELAT, J. P., 1992. Compared efficacy of repeated annual and semi-annual doses of ivermectin and diethylcarbamazine for prevention of Wuchereria bancrofti filariasis in French Polynesia. Final evaluation. Tropical Medicineand Parasitology, 43:91-94.

CDC (Centers for Disease Control), 1993. Recommendations of the International Task Force for Disease Eradication. Morbidity and Mortality Weekly Report, 42:1-38.

CHAN, M. S.; GUYAT, H. L.; BUNDY, D. A.; BOOTH, M.; FULFORD, A. J. \& MEDLEY, G. F., 1995. The development of an age structured model for schistosomiasis transmission dynamics and control and its validation for Schistosoma mansoni. Epidemiology and Infections, 115:325-344.

CHANTEAU, S.; LUQUIAUD, P.; FAILLOUX, A. \& WILLIAMS, S. A., 1994. PCR-based detection of Wuchereria bancrofti larvae in pools of mosquitoes. Transactions of the Royal Society of Tropical Medicine and Hygiene, 88:665-666.

CHEONG, W. C. \&YAP, H. H., 1985. Bioassay of Bacillus sphaericus (strain 1953) against mosquitoes of public health importance in Malaysia. Southeast Asian Journal of Tropical Medicine and Public Health, 16:54-58.

CHODAKEWITZ, J., 1995. Ivermectin and lymphatic filariasis: A clinical update. Parasitology Today, 11:233-235.

COUTINHO, A.; DREYER, G.; MEDEIROS, Z.; LOPES, E.; MACHADO, G.; GALDINO, E.; RIZZO, J. A.; ANDRADE, L. D.; SANTOS, A.; MOURA, I.; GODOY, J. \& OTTESEN, E. A., 1994. Ivermectin treatment of bancroftian filariasis in Recife, Brazil. American Journal of Tropical Medicineand Hygiene, 50:339348.

DREYER, G.; OTTESEN, E. A.; GALDINO, E.; ANDRADE, L.; ROCHA., A.; MEDEIROS, Z.; MOURA, I.; CASSIMIRO, M. I.; BÉLIZ, M. F. \& COUTINHO, A., 1992. Renal abnormalities in microfilaremic patients with bancroftian filariasis. American Journal of Tropical Medicineand Hygiene, 46:745-751.
DREYER, G.; PIRES, M. L.; ANDRADE, L. D.; LOPES, E.; MEDEIROS, Z.; TENORIO, J.; COUTINHO, A.; NORÕES, J. \& FIGUEIREDO-SILVA, J., 1994. Tolerance of diethylcarbamazine by microfilaremic and amicrofilaremic individuals in an endemic area of bancroftian filariasis, Recife, Brazil. Transactions of the Royal Society of Tropical Medicine and Hygiene, 88:232-236.

DREYER, G.; AM ARAL, F.; NOR'ES, J.; MEDEIROS, Z. $\&$ ADDISS, D., 1995. A New tool to assess the adulticidal efficacy in vivo of antifilarial drugs for bancroftian filariasis. Transactions of the Royal Society of Tropical Medicine and Hygiene, 89:255256.

DREYER, G.; COUTINHO, A.; MIRANDA, D.; NORÕES, J.; RIZZO, J. A.; GALDINO, E.; ROCHA, A.; MEDEIROS, Z.; ANDRADE, L. D.; SANTOS, A.; FIGUEREDO-SILVA, J. \& OTTESEN, E.A., 1995b. Treatment of bancroftian filariasis in Recife, Brazil: a two year comparative study of the efficacy of single treatments with ivermectin or diethylcarbamazine. Transactions of the Royal Society of Tropical Medicineand Hygiene, 89:98-102.

DREYER, G.; NORÕES, J.; AM ARAL, F.; NEN, A.; MEDEIROS, Z.; COUTINHO, A. \& ADDISS, D., 1995c. Direct assessment of the adulticidal efficacy of single dose ivermectin in bancroftian filariasis. Transactions of the Royal Society of Tropical Medicineand Hygiene, 89:441-443.

DREYER, G.; ADDISS, D.; NORÕES, J.; AM ARAL, F.; ROCHA, A. \& COUTINHO, A., 1996. Ultrasonographic assessment of the adulticidal efficacy of repeated high-dose ivermectin in bancroftian fiIariasis. Tropical Medicine and International Health, 1:427-432.

DREYER, G.; MEDEIROS, Z.; NETTO, M. J.; LEAL, N.; CASTRO, L. G. \& PIESSENS, W. F. Acute attacks in persons living in areas endemic for bancroftian filariasis: differentiation of two syndromes. Acta Tropica, no prelo.

FIGUEREDO-SILVA, J.; JUNGMANN, P.; NOR'ES, J.; PIESSENS, W. F.; COUTINHO, A.; BRITO, C.; ROCHA, A. \& DREYER, G., 1996. Histological evidence for the adulticidal effect of low doses of diethylcarbamazine in bancroftian filariasis. Transactions of the Royal Society of Tropical Medicine and Hygiene, 90:192-194.

GELBAND, H., 1994. Diethylcarbamazine salt in the control of lymphatic filariasis. American Journal of Tropical Medicineand Hygiene, 50:655-662.

HOUGARD, M. J.; MBENTENGAM, R.; LOCHOUARN, L.; ESCAFREE, H.; DARRIET, F.; BARBAZAN, P. \& QUILLÉVÉRÉ, D., 1993. Campaign against Culex quinquefasciatus using Bacillus sphaericus: Result of a pilot project in a large urban area of equatorial Africa. Bulletin of theWorld Health Organization, 71:367-375.

KAR, S. K.; MANIA, J. \& PATNAIK. S., 1994. The use of ivermectin for scabies. National Medical of India, 7:15-16.

MOULIA-PELAT, J. P.; GLAZIOU, P.; NGUYEN, L. N.; CHANTEAU, S.; PLICHART, R.; BEYLIER, I.; MARTIN, P. M. V. \& CARTEL, J. P., 1994. Ivermectin $400 \mu \mathrm{g} / \mathrm{Kg}$ : Long-term suppression of microfilari- 
ae in bacroftian filariasis. Transaction of the Royal Society of Tropical Medicineand Hygiene, 88:107109.

MOULIA-PELAT, J. P.; NGUYEN, L. N.; HASCOET, H.; LUQUIAUD, P. \& NICOLAS, L., 1995a. Advantages of an annual single dose of ivermectin 400 micrograms/ kg plus diethylcarbamazine for community treatment of bancroftian filariasis. Transactions of the Royal Society of Tropical Medicineand Hygiene, 89:682-682.

MOULIA-PELAT, J. P.; GLAZIOU, P.; WEIL, G. J.; GAXOTTE, P. \& NICOLAS, L., 1995b. Combination ivermectin plus diethylcarbamazine, a new effective tool for control of lymphatic filariasis. Tropical Medicineand Parasitology, 46:9-12.

NORÕES, J.; ADDISS, D.; AMARAL, F.; COUTINHO, A.; MEDEIROS, Z. \& DREYER, G., 1996a. Occurrence of living adult Wuchereria bancrofti in the scrotal area of men with microfilaremia. Transactions of the Royal Society of Tropical Medicine and Hygiene, 90:55-56.

NORÕES, J.; ADDISS, D.; SANTOS, A.; MEDEIROS, Z.; COUTINHO, A. \& DREYER, G., 1996b. Ultrasonographic evidence of abnormal lymphatic vessels in young men with adult Wuchereria bancrofti infection in the scrotal area. Journal of Urology, 156:409-412.

NORÕES, J.; DREYER, G.; SANTOS, A.; MENDES, V. G.; MEDEIROS, Z. \& ADDISS, D., 1997. Assessment of the efficacy of diethylcarbamazine on adult Wuchereria bancrofti in vivo. 1997. Transactions of the Royal Society of Tropical Medicineand Hygiene, 91:78-81.

OTTESEN, E. A., 1985. Efficacy of diethylcarbamazine in eradicating infection with lymphatic-dwelling filariae in humans. Reviews of Infectious Diseases, 7:341-356.

OTTESEN, E. A. \& CAM PBELL, W. C., 1994. Ivermectin in human medicine. Journal of Antimicrobial Chemotherapy, 34:195-203.

PLAISIER, A. P.; VAN OOTM ARSSEN, G. J.; HABBEMA, J. D. F.; REMME, J \& ALLEY, E. S., 1990. ONCHOSIM : a model and computer simulation programme for the transmission and control of onchocerciasis. Computer Methods and Programs in Biomedicine, 31:43-56.

RÉGIS, L.; SILVA-FILHA, M. H. L. H. M.; OLIVEIRA, C. M. F.; RIOS, E. M.; SILVA, S. B. \& FURTADO, A. 1995. Integrated control measures against Culex quinquefasciatus, the vector of filariasis in Recife. Memórias do Instituto Oswal do Cruz, 90:115-119.
RICHARDS, F. O.; EBERHARD, M. L.; BRYAN, R. T.; MCNEELY, D. F.; LAM MIE, P. J.; MCNEELY, M. B.; BERNARD, Y.; HIGHTOWER, A. W. \& SPENCER, H. C., 1991. Comparison of high dose ivermectin and diethylcarbamazine for activity against bancroftian filariasis in Haiti. American Journal of Tropical Medicineand Hygiene, 44:3-10.

SASA, M., 1976. The antifilariasis campaign: its history and future prospects. In: Human Filariasis. A global survey of Epidemiology and Control. (M. Sasa, ed.), pp. 3-11. Tokyo: University of Tokyo Press.

SUTANTO, I.; BOREHAM, P. F. L.; MUNAWAR, M.; PURNOMO, \& PARTONO, F., 1985. Adverse reactions to a single dose of diethylcarbamazine in patients with Brugia malayi infection in Riau Province, west Indonesia. Southeast Asian Journal of Tropical Medicine and Public Health, 16:395-400.

TDR NEWS, 1996. Four TDR diseases can be "eliminated". TDR News, 49:1-8.

VANAM AIL, P.; SUBRAM ANIAM, S.; DAS, P. K.; PANI, S. P.; RAJAGOPALAN, P. K.; BUNDY, D. A. P. \& GRENFELL, B. T., 1989. Estimation of age specific rates of acquisition and loss of Wuchereria bancrofti infection adult worms. Transactions of the Royal Society of Tropical Medicine and Hygiene, 83:689-693.

WHO (World Health Organization), 1994. Strategies for Contol of Lymphatic Filariasis Infection and Disease: Report of a WHO/CTD/TDR Consultative Meeting Helt at the Universiti Sains Malaysia. Penang: Tropical Disease Research, Control of Tropical Disease, Filariasis.

WHO (World Health Organization), 1992. Lymphatic filariasis: the disease and its control. Fifth Report of the Expert Committee on Filariasis. WHO Technical Report Series, 821:1-71.

ZHENG, H.; PIESSENS, W. F.; ZHENGHOU, T.; WENFANG, C.; SHIHAI, W.; SHIZHI, C.; YANGMING, Y.; LAIFENG, L.; XIAORUI, C. \& GENBAO, G., 1991. Efficacy of ivermectin for control of microfilaremia recurring after treatment with diethylcarbamazine. I. Clinical and parasitological observations. American Journal of Tropical Medicineand Hygiene, 45:168-174.

ZHONG, M.; MCCARTHY, J.; BIERWERT, L.; LIZOTTEWANIEWSKI, M.; CHANTEAU, S.; NUTM AN, T.; OTTESEN, E. A. \& WILLIAMS, S., 1996. A polymerase chain reaction assay for detection of the parasite Wuchereria bancrofti in human blood samples. American Journal of Tropical Medicine and Hygiene, 54:357-363. 